\title{
Cardioprotective effects of ulinastatin against isoproterenol-induced chronic heart failure through the PI3K-Akt, p38 MAPK and NF-кB pathways
}

\author{
LIN LI ${ }^{1}$, JIANHUA HAO $^{1}$, XIAN JIANG ${ }^{2}, \mathrm{PING} \mathrm{LI}^{1}$ and $\mathrm{HU} \mathrm{SEN}^{3}$ \\ Departments of ${ }^{1}$ Anesthesia and ${ }^{2}$ Hepatobiliary Surgery; ${ }^{3}$ Institute of Burns, The First Affiliated Hospital \\ of PLA General Hospital, Beijing 100048, P.R. China
}

Received October 22, 2016; Accepted August 22, 2017

DOI: $10.3892 / \mathrm{mmr} .2017 .7934$

\begin{abstract}
The purpose of the present study was to evaluate the cardioprotective effect of ulinastatin against isoproterenol-induced chronic heart failure $(\mathrm{CHF})$. Compared with the control group, treatment with ulinastatin decreased interventricular septal thickness and left ventricular posterior wall thickness, and improved the left ventricular ejection fraction, left ventricular fractional shortening and peak $\mathrm{E}$ and peak $\mathrm{A}$ ratio in the isoproterenol-induced $\mathrm{CHF}$ rat. In addition, ulinastatin suppressed inflammation, oxidative stress and apoptosis in heart tissue from isoproterenol-induced $\mathrm{CHF}$ rats. Ulinastatin induced the activation of the phosphatidylinositol 3-kinase (PI3K)/RAC- $\alpha$ serine/threonine protein kinase (Akt) signaling pathway and downregulated the p38 mitogen-activated protein kinase (MAPK) and nuclear factor $(\mathrm{NF})-\kappa \mathrm{B}$ pathway in isoproterenol-induced $\mathrm{CHF}$ rats. These data demonstrated the cardioprotective effect of ulinastatin against isoproterenol-induced chronic heart failure through the PI3K-Akt, p38 MAPK and NF-кB pathways.
\end{abstract}

\section{Introduction}

Chronic heart failure (CHF) occurs as a result of a number of cardiovascular diseases and is a complex pathophysiological process; neurohormonal disorders and ventricular remodeling are important pathophysiological changes for patients with CHF (1). A large dose (>85 mg/kg) of isoproterenol (ISO) may cause diffuse myocardial necrosis and fibrosis, which may gradually develop into dilated cardiomyopathy heart failure (2). ISO may cause myocardial necrosis similar to

Correspondence to: Dr Hu Sen, Institute of Burns, The First Affiliated Hospital of PLA General Hospital, 51 Fuchengmen Road, Haidian, Beijing 100048, P.R. China

E-mail: hmup6811725@126.com

Key words: ulinastatin, chronic heart failure, phosphatidylinositol 3-kinase, $\mathrm{RAC}-\alpha$ serine/threonine protein kinase, p38 mitogen-activated protein kinase, nuclear factor- $\kappa \mathrm{B}$ myocardial infarction, but it maintains effective coronary circulation (3). This necrosis depends on dose and time, and ranges between endocardial focal necrosis and transmural necrosis. There are multiple factors underlying the induction of myocardial necrosis by ISO, primarily associated with its cardiac toxicity, and a previous study demonstrated that the following factors are relevant: Relative hypoxia; microcirculation; permeability alterations in myocardial cell membranes; overload of $\mathrm{Ca}^{2+}$; toxic effects of ISO oxidation products; and myocardial ischemia-reperfusion injury (3).

$\mathrm{CHF}$ is the end result of the majority of cardiovascular diseases. Despite progress in treatment methods and an improved prognosis for CHF during the past two decades, the overall morbidity, mortality and readmission rates for heart failure remain high (4). At present, clinicians recognize that heart failure is an inflammatory reaction, and increasing evidence has demonstrated that inflammation serves an important role in the development of heart failure (5). Previous reports have indicated that pro-inflammatory cytokines are frequently overexpressed in patients with $\mathrm{CHF}$, including C-reactive protein, tumor necrosis factor- $\alpha$ (TNF- $\alpha$ ), interleukin (IL)-1, IL-6 and monocyte chemoattractant protein-1, which induce myocardial apoptosis and fibrosis, result in cardiac remodeling, and promote the development of CHF; these effects are positively correlated with the severity of heart failure, indicating a poor prognosis for patients with $\mathrm{CHF}(6,7)$. Therefore, anti-inflammatory treatment for heart failure may represent a novel approach.

A recent study demonstrated that for $\mathrm{CHF}$, in addition to enhanced sympathetic excitability and abnormal secretion of various humoral factors, overexpression of inflammatory cytokines and imbalances within the immune system are important aspects of its complex pathophysiology (8). The abnormal inflammatory response mediated by pro-inflammatory cytokines is associated with left ventricular remodeling, left ventricular failure, endothelial injury, myocardial apoptosis of endothelial cells and cachexia in patients with $\mathrm{CHF}$, and promotes the development of heart failure (9). Immune system disorders are associated with myocardial cell death, fibrosis, systolic dysfunction and deterioration, and heart failure severity (10).

The phosphatidylinositol 3-kinase (PI3K)/RAC- $\alpha$ serine/threonine protein kinase (Akt) signaling pathway is 
widely present in mammalian cells and serves a complex role (11). When CHF occurs, PI3K/Akt may achieve a cardioprotective effect by regulating downstream target genes (12).

Mitogen-activated protein kinase (MAPK) belongs to the family of serine/threonine protein kinases, which may be activated by certain ligands, including receptors, growth factors, G-protein-coupled receptors and certain stressors (13). p38 MAPK belongs to the same MAPK system as extracellular signal-regulated kinases $1 / 2$ and 5 , and c-Jun N-terminal kinase (JNK), and may be activated by stressors. JNK, p38 MAPK and extracellular signal-regulated kinase 5 are expressed in the human heart (14). The activities of JNK and p38 MAPK are markedly increased in CHF with ischemic cardiomyopathy (14). p38 MAPK are selectively expressed in myocardial cells of mice with myocardial infarction (15).

Ulinastatin is a type of glycoprotein, isolated and purified from the fresh urine of healthy adult males, which acts as a broad-spectrum enzyme inhibitor and may block the release of inflammatory cytokines, prevent the initiation of the cytokine cascade, suppress excessive activation of leukocytes, and block cycle of feedback activation among cytokines, inflammatory mediators and leukocytes (16). Previous studies have demonstrated, regarding the anti-inflammatory, immunomodulatory and visceral protective effects of ulinastatin, that ulinastatin exhibits a cellular protective function in ischemia-reperfusion injury in the liver, kidney, heart and lung, and improves immune function (16-18). The present study examined the hypothesis that the cardioprotective effect of ulinastatin may prevent ISO-induced $\mathrm{CHF}$, and aimed to elucidate the possible mechanism.

\section{Materials and methods}

Subjects, grouping and the CHF model. Male Sprague-Dawley rats (6-8 weeks old) weighing 200-220 g were purchased from Beijing Vital River Laboratory Animal Technology Co., Ltd. (Beijing, China) and caged individually at controlled temperature $\left(22-23^{\circ} \mathrm{C}\right)$ and humidity $(45-55 \%)$ with a 12 -hour light/dark cycle and free access to food, and water. All procedures were performed in accordance with the Guide for the Care and Use of Laboratory Animals and were approved by the medical Ethics Committee of the First Affiliated Hospital of PLA General Hospital (Beijing, China). A total of 30 male Sprague Dawley rats were randomly assigned to three groups: Control group $(n=6)$, ISO-induced CHF model $(n=20)$, and ulinastatin group $(n=20)$. In the control group, rats were intraperitoneally injected with normal saline. In the ISO-induced $\mathrm{CHF}$ model group, rats were intraperitoneally injected with $5 \mathrm{mg} / \mathrm{kg} / \mathrm{day}$ isoproterenol hydrochloride (Sigma-Aldrich; Merck KGaA, Darmstadt, Germany) for 5 days, and subsequently with normal saline for 5 days. In the ulinastatin group, ISO-induced CHF model rats were pretreated with a one off dose of 2,500 IU/kg ulinastatin (Sigma-Aldrich; Merck $\mathrm{KGaA}$ ) for 1 week, followed by treatment with $5 \mathrm{mg} / \mathrm{kg} /$ day isoproterenol hydrochloride (Sigma-Aldrich; Merck KGaA) for 5 days, and subsequently with normal saline for 5 days.

Echocardiographic assessment of heart function. Rats were anesthetized with $30 \mathrm{mg} / \mathrm{kg}$ pentobarbital sodium and an ultrasound probe was placed in the left sternal border.
Interventricular septal thickness (IVS) and left ventricular posterior wall thickness (LVPW), the left ventricular ejection fraction (LVEF), left ventricular fractional shortening (LVFS) and peak E to peak A ratio were obtained using the long axis of left ventricle and the maximum diameter, and calculated using ECToolbox ${ }^{\mathrm{TM}}$ for Xeleris ${ }^{\mathrm{TM}}$ version 2 software $(\mathrm{GE}$ Healthcare, Chicago, IL, USA).

Measurements. Rats were anesthetized with $30 \mathrm{mg} / \mathrm{kg}$ pentobarbital sodium and sacrificed by decollation. Peripheral blood was collected and serum was absorbed following centrifugation at 5,000 x g for $10 \mathrm{~min}$ at $4^{\circ} \mathrm{C}$. Subsequently, serum was used to measure NF- $\kappa \mathrm{B}$ (cat no. H202; Nanjing Jiancheng Biology Engineering Institute, Nanjing, China), TNF- $\alpha$ (cat no. PT51), IL-1 $\beta$ (cat no. PI303), IL-6 (cat no. PI328), glutathione peroxidase (GSH-PX; A005), glutathione (GSH; cat no. A005), superoxide dismutase (SOD; cat no. A001-3), malondialdehyde (MDA; cat no. A003-1), caspase-3 (cat no. C1115) and caspase-9 (cat no. C1157) (all from Beyotime Institute of Biotechnology, Haimen, China) using commercial kits. The optical density (OD) of NF- $\kappa$ B, TNF- $\alpha$, IL-1 $\beta$, IL-6, GSH-PX, GSH, SOD and MDA was measured using an ELX-800 microplate assay reader (BioTek Instruments, Inc., Winooski, VT, USA) at $450 \mathrm{~nm}$. The OD of caspase-3 and caspase-9 was measured using the ELX-800 microplate assay reader at $405 \mathrm{~nm}$.

Western blotting. Rats were anesthetized with $30 \mathrm{mg} / \mathrm{kg}$ pentobarbital sodium and sacrificed by decollation. Heart tissue samples were collected and washed with PBS. The frozen myocardial tissues were homogenized in tissue lysis buffer (radioimmunoprecipitation buffer; Beyotime Institute of Biotechnology) on ice for $20 \mathrm{~min}$. The supernatant was collected following centrifugation at 5,000 x $\mathrm{g}$ for $10 \mathrm{~min}$ at $4{ }^{\circ} \mathrm{C}$ and protein concentration was determined using a bicinchoninic acid kit (Beyotime Institute of Biotechnology). Protein samples (50-80 $\mu \mathrm{g})$ were subjected to SDS-PAGE on 6-10\% gels and transferred to a nitrocellulose membrane (EMD Millipore, Billerica, MA, USA). The membrane was blocked with $5 \%$ skim milk powder in Tris-buffered saline with Tween-20 (TBST) for $1 \mathrm{~h}$ at $37^{\circ} \mathrm{C}$ and probed with the following primary antibodies: Anti-p65 (cat no. 8242; 1:2,000; Cell Signaling Technology, Inc., Danvers, MA, USA), anti-apoptosis regulator Bcl-2 (Bcl-2; cat no. sc-783; 1:500; Santa Cruz Biotechnology, Inc., Dallas, TZ, USA), anti-apoptosis regulator BAX (Bax; cat no. 2772; 1:5,000), anti-Akt (cat no. 4685; 1:2,000), anti-phosphorylated (p)-Akt (cat no. 4060; 1:2,000), anti-p-p38 (cat no. 4511; 1:2,000) and anti-GAPDH (cat no. 5174) (all from Cell Signaling Technology, Inc.), at $4^{\circ} \mathrm{C}$ overnight. The membranes were washed with TBST and incubated with anti-rabbit horseradish peroxidase-conjugated secondary antibodies (cat no. sc-2030; 1:5,000; Santa Cruz Biotechnology, Inc.) for $1 \mathrm{~h}$ at $37^{\circ} \mathrm{C}$. Protein bands were visualized using the Enhanced Chemiluminescence Plus western blotting detection system (PerkinElmer, Inc., Waltham, MA, USA) and quantified using Image Studio version 1.1 software (LI-COR Biosciences, Lincoln, NE, USA).

Statistical analysis. Quantitative data are expressed as the mean \pm standard error of the mean $(n=3)$ using SPSS 

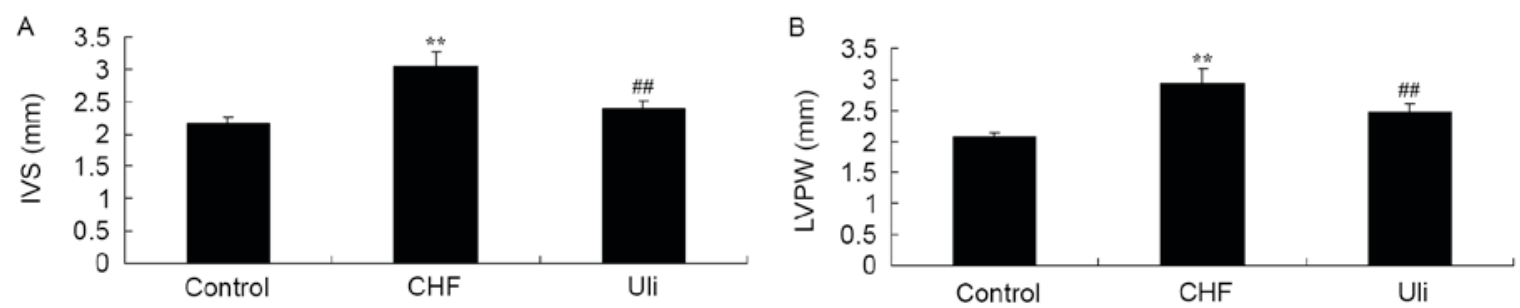

Figure 1. Cardioprotective effect of ulinastatin protects against alterations to the cardiac structure. The cardioprotective effect of ulinastatin protected against (A) IVS and (B) LVPW. ${ }^{* *} \mathrm{P}<0.01$ vs. control; "\# $\mathrm{P}<0.01$ vs. CHF. CHF, isoproterenol-induced chronic heart failure model group; Uli, ulinastatin group; IVS, interventricular septal thickness; LVPW, left ventricular posterior wall thickness.
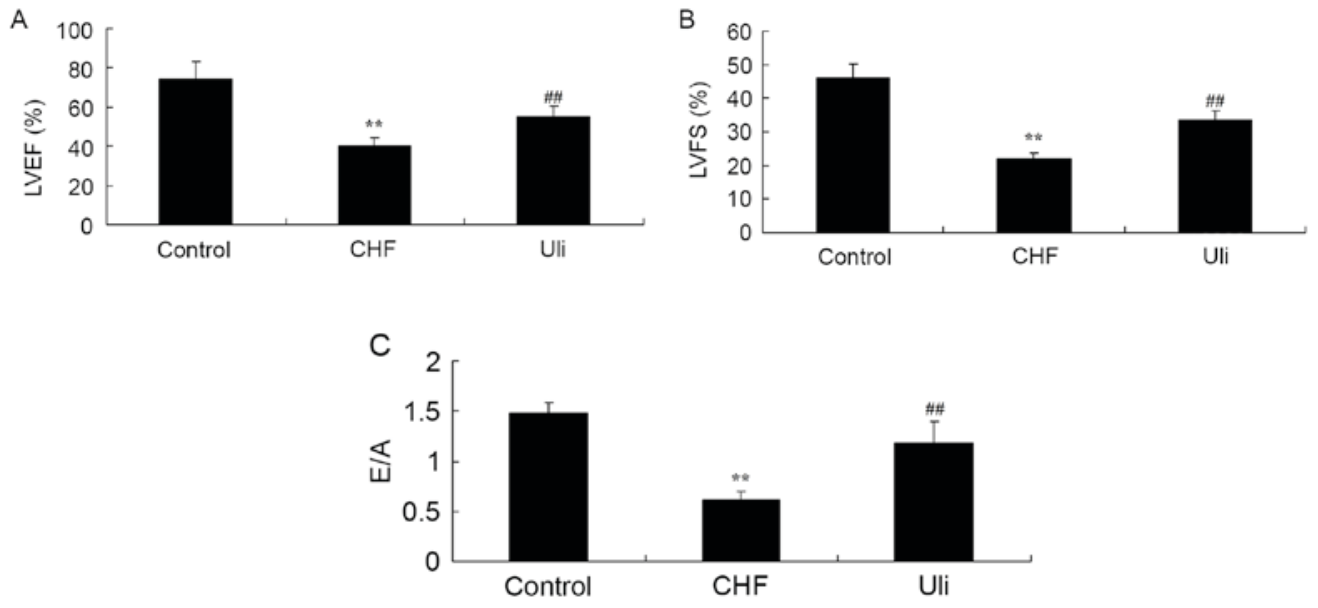

Figure 2. Cardioprotective effect of ulinastatin on cardiac function. The cardioprotective effect of ulinastatin protected against (A) LVEF, (B) LVFS and (C) peak E/A ratio. ${ }^{* *} \mathrm{P}<0.01$ vs. control; ${ }^{\# \#} \mathrm{P}<0.01$ vs. CHF. CHF, isoproterenol-induced chronic heart failure model group; Uli, ulinastatin group; LVEF, left ventricular ejection fraction; LVFS, left ventricular fractional shortening.

(version 17.0; SPSS, Inc., Chicago, IL, USA). Data were analyzed using one-way analysis of variance followed by Tukey's post hoc test. $\mathrm{P}<0.05$ was considered to indicate a statistically significant difference.

\section{Results}

Cardioprotective effect of ulinastatin against alterations in cardiac structure. At week 5, the IVS and LVPW of the ISO-induced CHF model animals were increased compared with those of the control group (Fig. 1). Treatment with Ulinastatin prevented IVS and LVPW in ISO-induced CHF rat, compared with the ISO-induced CHF model rat group (Fig. 1).

Cardioprotective effect of ulinastatin on cardiac function. At week 5, the data presented in Fig. 2 revealed significant inhibitions of LVEF, LVFS and peak E/A ratio in the ISO-induced CHF model rat, compared with the control group. Treatment with ulinastatin significantly increased LVEF, LVFS and peak E/A ratio in the ISO-induced CHF rat, compared with ISO-induced CHF model rat group (Fig. 2).

Cardioprotective effect of ulinastatin against inflammatory factors. In order to examine the cardioprotective effect of ulinastatin against inflammatory factors, NF- $\mathrm{kB}, \mathrm{TNF}-\alpha$, IL- $1 \beta$ and IL- 6 expression levels in the ISO-induced CHF

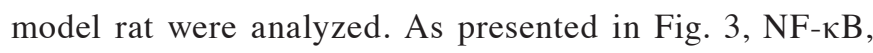
TNF- $\alpha$, IL- $1 \beta$ and IL- 6 levels in the ISO-induced CHF model rat were significantly increased compared with the control group. Ulinastatin significantly decreased NF- $\kappa B$, TNF- $\alpha$, IL- $1 \beta$ and IL- 6 levels in the ISO-induced CHF model rat, compared with the ISO-induced CHF model group (Fig. 3).

Cardioprotective effect of ulinastatin against $N F-\kappa B$ signaling pathway activation and an increased $\mathrm{Bax} / \mathrm{Bcl}-2$ ratio. In order to further examine the mechanism underlying the anti-inflammatory effect of ulinastatin, the NF- $\kappa B$ pathway and the expression of $\mathrm{Bax} / \mathrm{Bcl}-2$ were analyzed in the present study. The data presented in Fig. 4 demonstrated that NF- $\mathrm{KB}$ protein expression and the $\mathrm{Bax} / \mathrm{Bcl}-2$ ratio were significantly upregulated in ISO-induced CHF model rats, compared with the control group. Treatment with ulinastatin significantly suppressed NF- $\mathrm{KB}$ protein expression and the Bax/Bcl-2 ratio in ISO-induced CHF rats, compared with the ISO-induced CHF model group (Fig. 4).

Cardioprotective effect of ulinastatin against oxidative stress. The effects of ulinastatin on the expression levels of CHF, GSH-PX, GSH, SOD and MDA in ISO-induced CHF rats were measured in the present study. Fig. 5 illustrates that the levels of GSH-PX, GSH and SOD were significantly decreased and the MDA level was significantly increased in the ISO-induced CHF rat, compared with the control group. In ISO-induced 

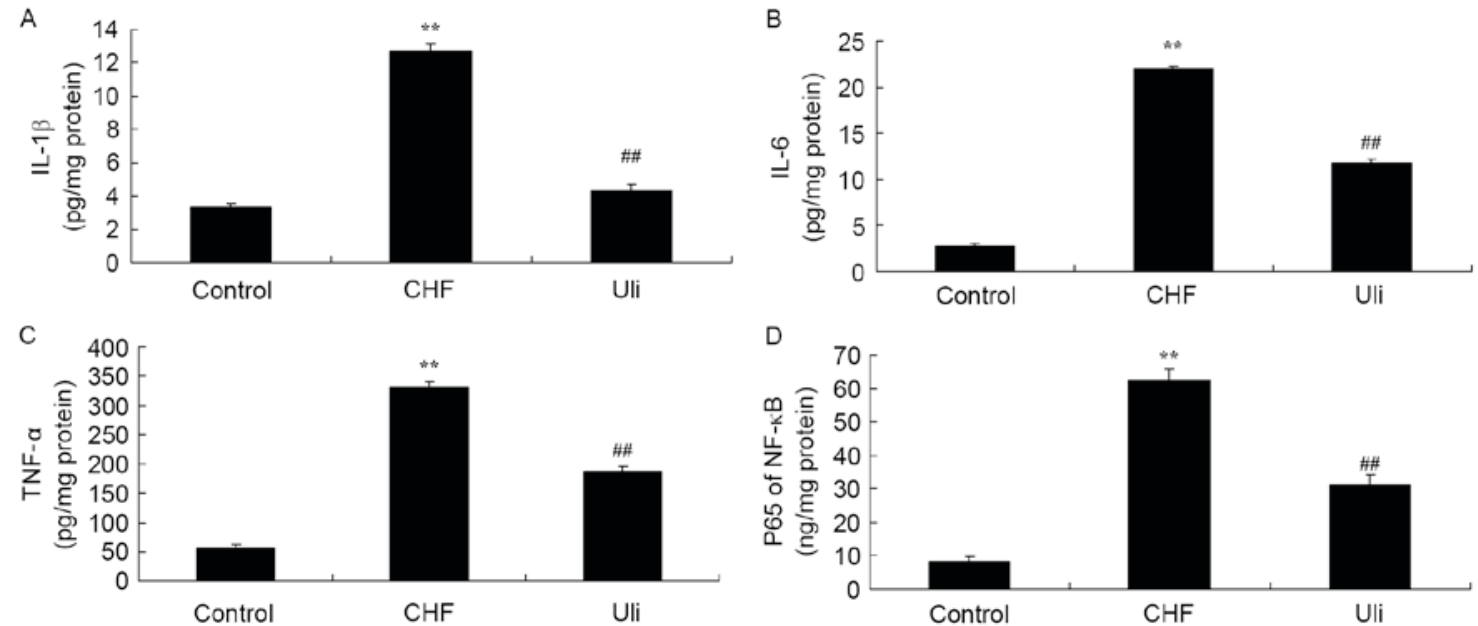

Figure 3. Cardioprotective effect of ulinastatin against inflammatory factors. The cardioprotective effect of ulinastatin protected against increases in (A) IL-1 $\beta$, (B) IL-6, (C) TNF- $\alpha$ and (D) NF- $\kappa B$. ${ }^{* *} \mathrm{P}<0.01$ vs. control; "\# $\mathrm{P}<0.01$ vs. CHF. CHF, isoproterenol-induced chronic heart failure model group; Uli, ulinastatin group; IL, interleukin; TNF- $\alpha$, tumor necrosis factor- $\alpha$; NF- $\kappa \mathrm{B}$, nuclear factor- $\kappa \mathrm{B}$.
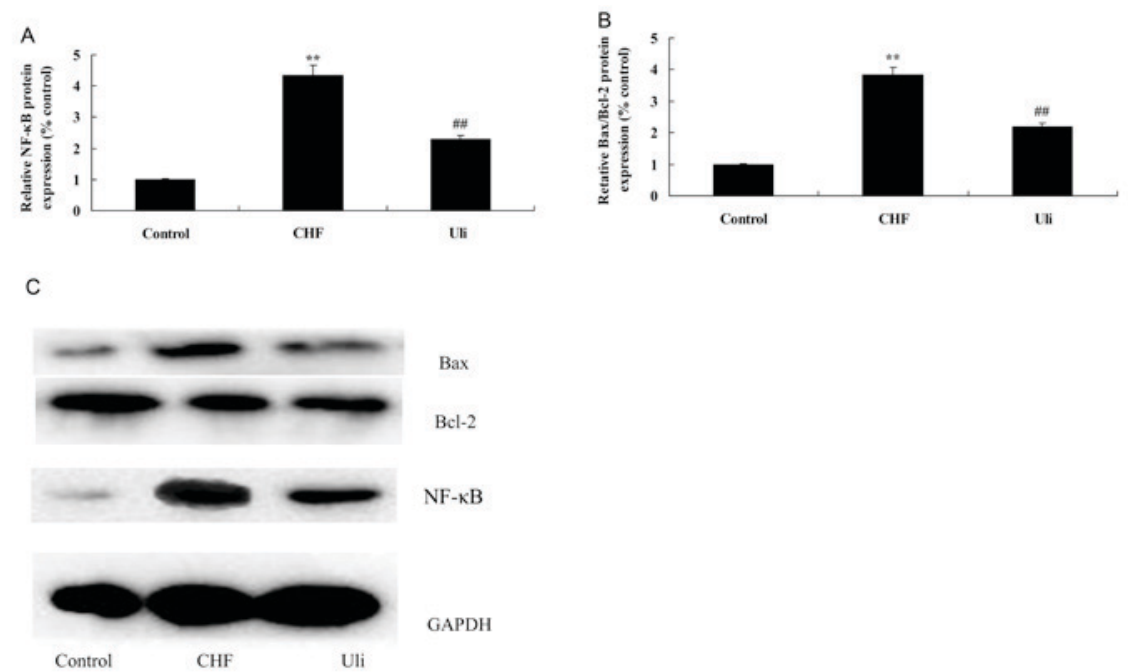

Figure 4. Cardioprotective effect of ulinastatin against NF- $\mathrm{kB}$ signaling pathway activation and increased Bax/Bcl-2 expression. The cardioprotective effect of ulinastatin protected against (A) NF- $\mathrm{kB}$ protein expression and (B) an increase in the Bax/Bcl-2 ratio. (C) Representative image of western blot. ${ }^{* *} \mathrm{P}<0.01$

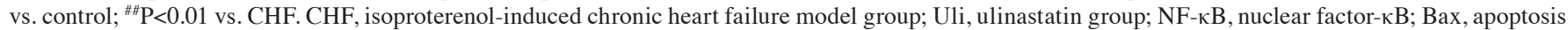
regulator $\mathrm{BAX}$; $\mathrm{Bcl}-2$, apoptosis regulator $\mathrm{Bcl}-2$.

CHF rats treated with ulinastatin, a significant increase in GSH-PX, GSH and SOD levels, and an inhibition of MDA, were observed compared with the ISO-induced CHF model group (Fig. 5).

Cardioprotective effect of ulinastatin against caspase-3 and caspase-9 activity. The expression of caspase- 3 and caspase- 9 was analyzed using commercial kits, in order to further examine the effect of ulinastatin on apoptosis in CHF. As presented in Fig. 6, significant increases in caspase-3 and caspase-9 expression were observed in the ISO-induced CHF rat model group, compared with the control group. Following treatment with isoproterenol hydrochloride for 5 days, ulinastatin significantly inhibited the expression of caspase-3 and caspase- 9 in ISO-induced CHF rats, compared with the ISO-induced CHF model group (Fig. 6).
Cardioprotective effect of ulinastatin against an increased $p$-Akt/Akt ratio and p-38 expression. In order to assay the alteration in $\mathrm{p}$-Akt/Akt protein expression in heart tissue samples, western blotting was performed on cardiac tissues from each group. As presented in Fig. 7, the p-Akt/Akt ratio was decreased and p-38 protein expression of the ISO-induced $\mathrm{CHF}$ model was increased compared with the control group. Pretreatment with ulinastatin significantly inhibited the alterations in the $\mathrm{p}$-Akt/Akt ratio and $\mathrm{p}-38$ protein expression in ISO-induced $\mathrm{CHF}$ rats, compared with the ISO-induced $\mathrm{CHF}$ model group (Fig. 7).

\section{Discussion}

CHF is the most severe form of cardiovascular disease (19). Due to improvements in medical technology, the incidences 

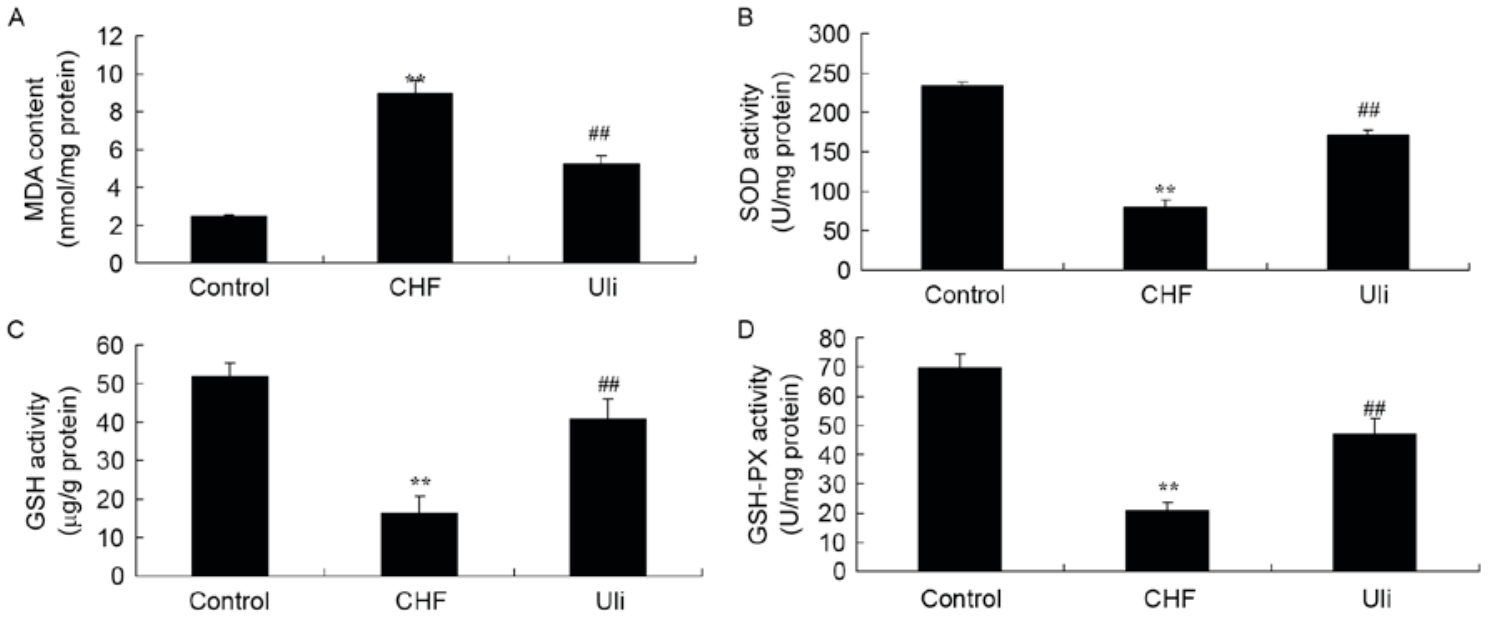

Figure 5. Cardioprotective effect of ulinastatin against oxidative stress. The cardioprotective effect of ulinastatin protected against increased (A) MDA, (B) SOD, (C) GSH and (D) GSH-PX levels. ${ }^{* *} \mathrm{P}<0.01$ vs. control; ${ }^{\#} \mathrm{P}<0.01$ vs. CHF. CHF, isoproterenol-induced chronic heart failure model group; Uli, ulinastatin group; MDA, malondialdehyde; SOD, superoxide dismutase; GSH, glutathione; GSH-PX, glutathione peroxidase.
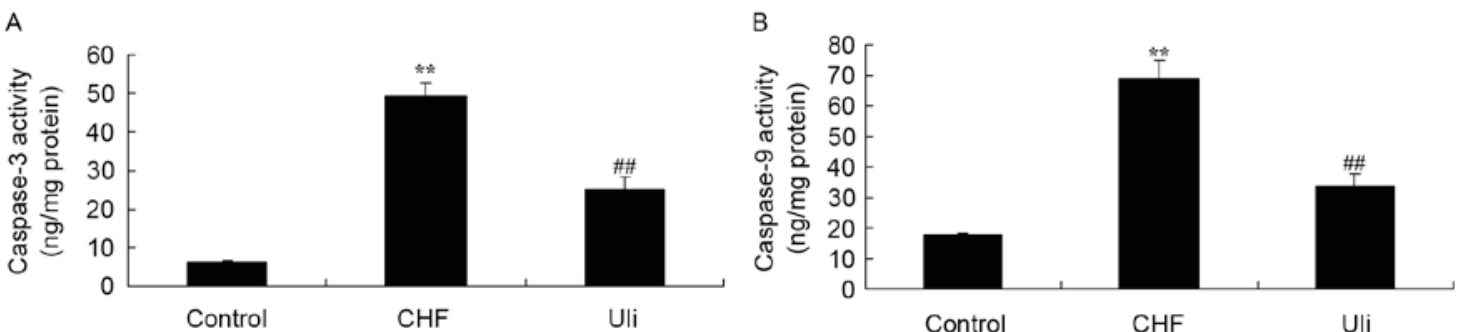

Figure 6. Cardioprotective effect of ulinastatin against caspase-3 and caspase- 9 activity. The cardioprotective effect of ulinastatin protected against increased (A) caspase-3 (A) and (B) caspase-9 activity. ${ }^{* *} \mathrm{P}<0.01$ vs. control; ${ }^{\# \#} \mathrm{P}<0.01$ vs. CHF. CHF, isoproterenol-induced chronic heart failure model group; Uli, ulinastatin group.
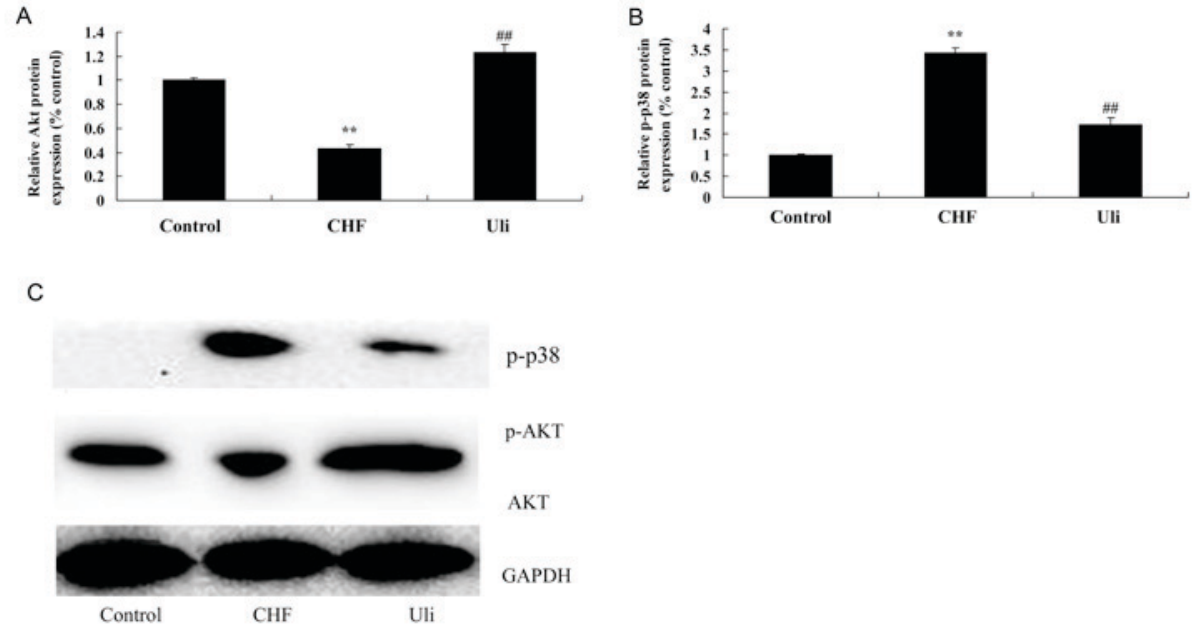

Figure 7. Cardioprotective effect of ulinastatin against decreased p-Akt and increased p38 protein expression. The cardioprotective effect of ulinastatin protected against (A) decreased p-Akt and (B) increased $\mathrm{p} 38$ protein expression, as demonstrated by statistical analysis and $(\mathrm{C})$ western blotting. ${ }^{* *} \mathrm{P}<0.01 \mathrm{vs}$. control; ${ }^{\# \#} \mathrm{P}<0.01$ vs. CHF. CHF, isoproterenol-induced chronic heart failure model group; Uli, ulinastatin group. p, phosphorylated; Akt, RAC- $\alpha$ serine/threonine protein kinase; 338 , p38 mitogen-activated protein kinase.

of other cardiovascular diseases are decreasing; however the incidence of CHF exhibits the opposite trend, and the World Health Organization has established the pathogenesis and treatment of heart failure as the focus of the prevention and treatment of cardiovascular diseases (20,21). A previous study has demonstrated that, in the pathogenesis of CHF, the NF- $\mathrm{kB}$ signal transduction pathway is associated with apoptosis and myocardial remodeling, which serve important roles (22). 
The results of the present study demonstrated that ulinastatin prevented the increase in IVS and LVPW, and increased LVEF and LVFS in ISO-induced CHF rats.

As a transcription factor, $\mathrm{NF}-\kappa \mathrm{B}$ is able to regulate the immune response, including effects on innate immunity and acquired immunity, which regulates the expressions of a series of inflammatory cytokines and serves an important role in the pathogenesis of CHF (23). During CHF compensation to decompensation, the amount of cytokines released has been demonstrated to be increased compared with sham controls, and is positively-associated with the activation of NF- $\kappa \mathrm{B}$, suggesting that the generation and activation of cytokines may serve a regulatory role through the activation of $\mathrm{NF}-\kappa \mathrm{B}(24,25)$. The results of the present study demonstrated that ulinastatin significantly inhibited NF- $\kappa$ B, TNF- $\alpha$, IL- $1 \beta$ and IL- 6 levels in ISO-induced $\mathrm{CHF}$ rats through suppression of NF- $\kappa$. Hou et al (16) posited that ulinastatin may inhibit inflammation via regulation of the 5 -AMP-activated protein kinase/NF- $\kappa \mathrm{B}$ pathway in lipopolysaccharide-induced acute lung injury in mice.

$\mathrm{CHF}$ is the final result of a number of cardiovascular diseases; the pathogenesis of heart failure is very complex, and involves apoptosis, inflammation, myocardial remodeling and mitochondrial injury of nerve-humoral factors regulating system are interacted with one another. In addition, the associated cell signal transduction mechanisms are complex (26). The PI3K/Akt signaling pathway serves an important role the regulation of a series of myocardial protective functions, including myocardial cell survival, apoptosis, myocardial remodeling and inflammation, in the pathogenesis of heart failure (27). A previous study has confirmed that there is an important response relationship between the phosphorylation of Akt and myocardial protection (27). Pathological cardiac hypertrophy is an important indicator of the development of heart failure (28). Excessive cardiac hypertrophy results in heart compliance, decreased contractility, myocardial fibrosis and other irreversible alterations (29). Therefore, the inhibition of pathological cardiac hypertrophy and the delay in ventricular remodeling is an important potential strategy for the prevention and treatment of CHF (29). Protein kinase mTOR and endothelial nitric oxide synthase (eNOS) are considered to be the important regulators of pathological myocardial hypertrophy (27). In the present study, it was observed that ulinastatin significantly decreased the $\mathrm{p}$-Akt/Akt ratio in ISO-induced CHF rats.

Myocardial apoptosis is an important mechanism in the pathogenesis of heart failure, and is considered to be the threshold at which heart failure develops into decompensation. The anti-apoptotic effect mediated by the PI3K/Akt signaling pathway is of importance in the regulation of myocardial cellular apoptosis (15). The PI3K/Akt signal transduction pathway exerts its anti-apoptotic effect through the regulation of its downstream target proteins, including glycogen synthase kinase- $3 \beta$, caspase family proteins, apoptosis regulator Bcl-2 family proteins and eNOS (30). The results of the present study demonstrated that ulinastatin significantly suppressed the $\mathrm{Bax} / \mathrm{Bcl}-2$ ratio in ISO-induced $\mathrm{CHF}$ rats via the $\mathrm{PI} 3 \mathrm{~K} / \mathrm{Akt}$ signaling pathway. Kim et al (31) reported that ulinastatin exerted a protective effect against regional myocardial I/R injury through activation of PI3K-Akt signal transduction and inhibition of p38 MAPK.
Cardiac remodeling is an underlying process in heart failure, which includes remodeling associated with organizational structural alterations in myocardial cells, the extracellular matrix and the collagen fiber network, for example, during the processes of heart chamber expansion and ventricular hypertrophy, in addition to electrical remodeling associated with a variety of signal pathway alterations (32). Studies have demonstrated that p38 MAPK may be activated in the process of heart failure development, involved in the process of ventricular remodeling, and that the inhibition of p38 MAPK is beneficial for the improvement of ventricular remodeling $(14,33)$. During treatment of cardiac remodeling, p38 MAPK activity has been demonstrated to be inhibited, suggesting that p38 MAPK activation serves a positive role in the induction of cardiac remodeling fibrosis (33). The results of the present study indicated that ulinastatin significantly suppressed p-p-38 protein expression in ISO-induced CHF rats. Liu et al (34) reported that ulinastatin protected against lung injury via the p38 signaling pathway.

In the present study, it was observed that the cardioprotective effect of ulinastatin protected against ISO-induced $\mathrm{CHF}$, inflammation, oxidative stress and apoptosis via the PI3K-Akt, p38 MAPK and NF- $\mathrm{NB}$ pathways. Inhibiting inflammation, oxidative stress and apoptosis in ISO-induced CHF with ulinastatin, and illustrating its effect in decelerating the progression of cardiac remodeling requires further investigation.

\section{References}

1. Kitzman DW, Brubaker P, Morgan T, Haykowsky M, Hundley G, Kraus WE, Eggebeen J and Nicklas BJ: Effect of caloric restriction or aerobic exercise training on peak oxygen consumption and quality of life in obese older patients with heart failure with preserved ejection fraction: A randomized clinical trial. JAMA 315: 36-46, 2016.

2. Zhong M, Zhou H, Long C, Zhang Y, Cui W, Zhang H and Wang H: Natakalim ameliorates isoproterenol-induced chronic heart failure by protecting against endothelial dysfunction. Pharmacology 98: 99-110, 2016.

3. Zhang GX, Ohmori K, Nagai Y, Fujisawa Y, Nishiyama A, Abe Y and Kimura S: Role of AT1 receptor in isoproterenol-induced cardiac hypertrophy and oxidative stress in mice. J Mol Cell Cardiol 42: 804-811, 2007.

4. Sente T, Van Berendoncks AM, Jonckheere AI, Rodenburg RJ, Lauwers P, Van Hoof V, Wouters A, Lardon F, Hoymans VY and Vrints CJ: Primary skeletal muscle myoblasts from chronic heart failure patients exhibit loss of anti-inflammatory and proliferative activity. BMC Cardiovasc Disord 16: 107, 2016.

5. Cabassi A, Binno SM, Tedeschi S, Graiani G, Galizia C, Bianconcini M, Coghi P, Fellini F, Ruffini L, Govoni P, et al: Myeloperoxidase-related chlorination activity is positively associated with circulating ceruloplasmin in chronic heart failure patients: Relationship with neurohormonal, inflammatory and nutritional parameters. Biomed Res Int 2015: 691693, 2015.

6. Brouwers C, Kupper N, Pelle AJ, Szabó BM, Westerhuis BL and Denollet J: Depressive symptoms in outpatients with heart failure: Importance of inflammatory biomarkers, disease severity and personality. Psychol Health 29: 564-582, 2014.

7. Brouwers C, Mommersteeg PM, Nyklíček I, Pelle AJ, Westerhuis BL, Szabó BM and Denollet J: Positive affect dimensions and their association with inflammatory biomarkers in patients with chronic heart failure. Biol Psychol 92: 220-226, 2013.

8. Ranganathan P, Jayakumar C, Tang Y, Park KM, Teoh JP, Su H, Li J, Kim IM and Ramesh G: MicroRNA-150 deletion in mice protects kidney from myocardial infarction-induced acute kidney injury. Am J Physiol Renal Physiol 309: F551-F558, 2015.

9. Bodén S, Wennberg M, Van Guelpen B, Johansson I, Lindahl B, Andersson J, Shivappa N, Hebert JR and Nilsson LM: Dietary inflammatory index and risk of first myocardial infarction; a prospective population-based study. Nutr J 16: 21, 2017. 
10. D'Amario D, Cabral-Da-Silva MC, Zheng H, Fiorini C, Goichberg P, Steadman E, Ferreira-Martins J, Sanada F, Piccoli M,Cappetta D, et al: Insulin-like growth factor-1 receptor identifies a pool of human cardiac stem cells with superior therapeutic potential for myocardial regeneration. Circ Res 108: 1467-1481, 2011.

11. Hu LJ, Ren WY, Shen QJ, Ji HY and Zhu L: Inflammation in lung after acute myocardial infarction is induced by dendritic cell-mediated immune response. J Biol Regul Homeost Agents 31: 29-40, 2017.

12. Rocha JA, Ribeiro SP, França CM, Coelho O, Alves G, Lacchini S, Kallás EG, Irigoyen MC and Consolim-Colombo FM: Increase in cholinergic modulation with pyridostigmine induces anti-inflammatory cell recruitment soon after acute myocardia infarction in rats. Am J Physiol Regul Integr Comp Physiol 310: R697-R706, 2016.

13. Wu L, Mei L, Chong L, Huang Y, Li Y, Chu M and Yang X: Olmesartan ameliorates pressure overload-induced cardiac remodeling through inhibition of TAK1/p38 signaling in mice. Life Sci 145: 121-126, 2016.

14. Li CY, Zhou Q, Yang LC, Chen YH, Hou JW, Guo K, Wang YP and Li YG: Dual-specificity phosphatase 14 protects the heart from aortic banding-induced cardiac hypertrophy and dysfunction through inactivation of TAK1-P38MAPK/-JNK1/2 signaling pathway. Basic Res Cardiol 111: 19, 2016.

15. Cao Y, Ruan Y, Shen T, Huang X, Li M, Yu W, Zhu Y, Man Y, Wang S and Li J: Astragalus polysaccharide suppresses doxorubicin-induced cardiotoxicity by regulating the PI3k/Akt and p38MAPK pathways. Oxid Med Cell Longev 2014: 674219, 2014.

16. Hou S, Fang M, Zhu Q, Liu Y, Liu L and Li X: MicroRNA-939 governs vascular integrity and angiogenesis through targeting $\gamma$-catenin in endothelial cells. Biochem Biophys Res Commun 484: 27-33, 2017.

17. Häyry P, Aavik E and Myllärniemi M: Blockade of growth factor synthesis and growth factor action: Two possible sites of interference in allograft vessel disease and coronary bypass or balloon injury. Metabolism 45 (8 Suppl 1): S101-S103, 1996.

18. Delafontaine P, Song YH and Li Y: Expression, regulation, and function of IGF-1, IGF-1R, and IGF-1 binding proteins in blood vessels. Arterioscler Thromb Vasc Biol 24: 435-444, 2004.

19. Yang H, Zhang FF, Peng XH, Zhao DH and Peng J: Efficacy of medication directed by home-monitoring cardiac resynchronization therapy in chronic heart failure patients. Chin Med Sci J 29: 61-62, 2014.

20. Vergaro G, Prud'homme M, Fazal L, Merval R, Passino C, Emdin M, Samuel JL, Cohen Solal A and Delcayre C: Inhibition of galectin-3 pathway prevents isoproterenol-induced left ventricular dysfunction and fibrosis in mice. Hypertension 67: 606-612, 2016

21. Pitt B, Anker SD, Böhm M, Gheorghiade M, Køber L, Krum H, Maggioni AP, Ponikowski P, Voors AA, Zannad F, et al: Rationale and design of MinerAlocorticoid Receptor antagonist Tolerability Study-Heart Failure (ARTS-HF): A randomized study of finerenone vs. Eplerenone in patients who have worsening chronic heart failure with diabetes and/or chronic kidney disease. Eur J Heart Fail 17: 224-232, 2015.

22. Javan H, Szucsik AM, Li L, Schaaf CL, Salama ME and Selzman CH: Cardiomyocyte p65 nuclear factor- $\kappa \mathrm{B}$ is necessary for compensatory adaptation to pressure overload. Circ Heart Fail 8: 109-118, 2015 .
23. Cao W, Chen J, Chen Y, Chen S, Chen X, Huang H and Liu P: Advanced glycation end products induced immune maturation of dendritic cells controls heart failure through NF- $\mathrm{NB}$ signaling pathway. Arch Biochem Biophys 580: 112-120, 2015.

24. Killeen MJ, Linder M, Pontoniere P and Crea R: NF- $\kappa \beta$ signaling and chronic inflammatory diseases: Exploring the potential of natural products to drive new therapeutic opportunities. Drug Discov Today 19: 373-378, 2014.

25. Maier HJ, Schips TG, Wietelmann A, Krüger M, Brunner C, Sauter M, Klingel K, Böttger T, Braun T and Wirth $\mathrm{T}$ : Cardiomyocyte-specific I $\kappa \mathrm{B}$ kinase $(\mathrm{IKK}) / \mathrm{NF}-\kappa \mathrm{B}$ activation induces reversible inflammatory cardiomyopathy and heart failure. Proc Natl Acad Sci USA 109: 11794-11799, 2012.

26. Siman FD, Silveira EA, Fernandes AA, Stefanon I, Vassallo DV and Padilha AS: Ouabain induces nitric oxide release by a PI3K/Akt-dependent pathway in isolated aortic rings from rats with heart failure. J Cardiovasc Pharmacol 65: 28-38, 2015.

27. Cui J, Zhang F, Wang Y, Liu J, Ming X, Hou J, Lv B, Fang S and Yu B: Macrophage migration inhibitory factor promotes cardiac stem cell proliferation and endothelial differentiation through the activation of the PI3K/Akt/mTOR and AMPK pathways. Int J Mol Med 37: 1299-1309, 2016.

28. Sagara S, Osanai T, Itoh T, Izumiyama K, Shibutani S, Hanada K, Yokoyama $\mathrm{H}$, Yamamoto $\mathrm{Y}$, Yokota $\mathrm{T}$, Tomita $\mathrm{H}$, et al: Overexpression of coupling factor 6 attenuates exercise-induced physiological cardiac hypertrophy by inhibiting PI3K/Akt signaling in mice. J Hypertens 30: 778-786, 2012.

29. Sun GW, Qiu ZD, Wang WN, Sui X and Sui DJ: Flavonoids extraction from propolis attenuates pathological cardiac hypertrophy through PI3K/AKT signaling pathway. Evid Based Complement Alternat Med 2016: 6281376, 2016.

30. He SF, Jin SY, Wu H, Wang B, Wu YX, Zhang SJ, Irwin MG, Wong TM and Zhang Y: Morphine preconditioning confers cardioprotection in doxorubicin-induced failing rat hearts via ERK/GSK-3 $\beta$ pathway independent of PI3K/Akt. Toxicol Appl Pharmacol 288: 349-358, 2015.

31. Kim SJ, Yoo KY, Jeong CW, Kim WM, Lee HK, Bae HB, Kwak SH, Li M and Lee J: Urinary trypsin inhibitors afford cardioprotective effects through activation of PI3K-Akt and ERK signal transduction and inhibition of p38 MAPK and JNK. Cardiology 114: 264-270, 2009.

32. Gaur M, Ritner C, Sievers R, Pedersen A, Prasad M, Bernstein HS and Yeghiazarians Y: Timed inhibition of p38MAPK directs accelerated differentiation of human embryonic stem cells into cardiomyocytes. Cytotherapy 12: 807-817, 2010.

33. Sucharov CC: Role of p38MAPK in beta(2)AR-induced cardiomyopathy: At the heart of the matter? Future Cardiol 3: 387-389, 2007.

34. Liu J, Xiao X, Shen Y, Chen L, Xu C, Zhao H, Wu Y, Zhang Q, Zhong J, Tang Z, et al: MicroRNA-32 promotes calcification in vascular smooth muscle cells: Implications as a novel marker for coronary artery calcification. PLoS One 12: e0174138, 2017. 